\title{
Cutaneous/Subcutaneous Implantation of Endometriotic Tissue Following Surgery: A Case Report
}

\author{
Emily Christine D'Silva, Noor KhairiyahBinti Mustafa and Wan Ahmad Hazim Wan Ghazali* \\ Department of Obstetrics and Gynecology, Putrajaya Hospital, Malaysia
}

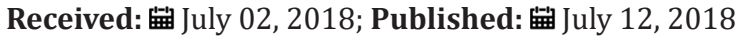

*Corresponding author: Wan Ahmad Hazim Wan Ghazali, Putrajaya Hospital, Pusat Pentadbiran Kerajaan Persekutuan, Presint 7 , 62250 Putrajaya, Malaysia

\begin{abstract}
Background: Scar endometriosis is a rare disease and poses a great challenge in diagnosis and thus its management.

Case: We present in this report a 32years old lady with underlying severe endometriosis who presented with persistent discharge and pain over her midline surgical scar site after two previous laparotomies. She was treated for wound breakdown which failed to resolve despite multiple courses of antibiotics and wound dressing. Surgical excision of the scar was done and sent for histopathology which confirms scar site endometriosis.

Result: Scar endometriosis should be suspected in women presenting with recurrent pain or discharge at an abdominal incision site.

Conclusion: Surgical excision should be the primary treatment with hormonal suppression as adjunct therapy.

Keywords: Scar Endometriosis; Cutaneous Endometriosis; Surgical Scar Endometriosis
\end{abstract}

\section{Introduction}

The presence of endometrial-like tissue outside the uterus, also known as endometriosis, induces a chronic, inflammatory that may affect $6-10 \%$ of total number of women[1,2].About $25 \%$ women with endometriosis are asymptomatic, while the majority may experience painful symptoms and or infertility. Nearly half of those affected women have chronic pelvic pain, while $70 \%$ present with dysmenorrheal [3].

Diagnosis of endometriosis is based on the women's history, signs and symptoms. It is supported by physical examination and imaging techniques. However, histopathological evidence of either from a direct biopsy of a lesion or from the tissue collected during surgery is required to confirm the diagnosis. The aetiology of endometriosis is not entirely clear. Structures that are close to the uterus within the pelvic cavity are commonly affected. However, in rare cases endometriotic tissue may also be implanted to other parts of the body including surgical scar sites following an abdominal gynaecological surgery.Endometriosis outside the pelvis occurs in about $12 \%$ of women with endometriosis[4].

Scar endometriosis, also known as cutaneous/subcutaneous endometriosis is a rare disease, and poses a great challenge in diagnosis. It occurs at surgical scar from abdominal or pelvic procedures including hysterectomy, Caesarean sections, episiotomy, and laparoscopy $[5,6]$. The prevalence has been estimated to be only $0.03 \%$ to $0.15 \%$ of all cases of endometriosis. The most accepted theory for the etiology of scar endometriosis is the iatrogenic transplantation of endometrial implants to the wound edge during an abdominal or pelvic surgery[7,8].The symptoms of scar endometriosis are nonspecific, classically involving abdominal wall pain or discomfort over the lesion which becomes more prominent especially at the time of menstruation. It may also be associated with swelling and slight bleeding or discharge from the lesion. Just like any other endometriosis, the diagnosis of scar endometriosis can only be confirmed by histopathological examination of the excised diseased tissue. The following is a case report of a patient with a lower midline scar following two gynaecological laparotomies, presented with scar endometriosis. The pathogenesis, differential diagnosis and treatment of this condition are discussed.

\section{Case Report}

A 32 years old Malay lady, nulliparous, married for 6 years was referred by a fertility specialist from another tertiary centre for laparoscopic bilateral salphingectomy and excision of stitch granuloma over previous incision site.She first presented 5 years ago in 2011 with infertility and underwent diagnostic laparoscopy, which was converted to laparotomy due to severe dense adhesions intra-operatively. A left endometrioma with bilateral hydrosalphinx were noted. Both ovaries and tubes were adhered to the uterus. Evisceration of left endometrioma was done and confirmed by histopathology. She was diagnosed with severe endometriosis and was treated with gonadotropins releasing hormones ( $\mathrm{GnRH}$ ) analogues for 6 months post-operatively. She was well for the next 
3 years until 2014 when she presented to a private medical centre with complaints of prolonged fever for two weeks associated with lower abdominal pain. Imaging studies noted massive bilateral hydrosalphinx. An urgent exploratory laparotomy was done after commencing broad-spectrum antibiotics. Large amount of pus was drained.

However bilateral salpingectomy was deferred due to friable tissue, which tends to bleed on touch. Unfortunately she underwent re-laparotomy during the same admission due to re-accumulation of pus in the pelvic cavity. 2 weeks after the surgery, she started experiencing discharge and intermittent discomfort from the surgical wound. She was treated for surgical wound breakdown with multiple courses of antibiotics and normal saline dressing. Nevertheless, symptoms did not improve and she notices the discharge was more periodic and prominent especially during menstruation. There was brownish discharge from the lesion. She was then treated with short trial of hormonal suppression with oral Dienogest. This improved her symptoms but recurred with cessation of treatment. A collection was detected by the ultrasound scan underneath the dark brown lesion over the scar site and a preliminary diagnosis of stitch granuloma was made with a differential diagnosis of possible scar endometriosis. She was then referred to our centre for further management.

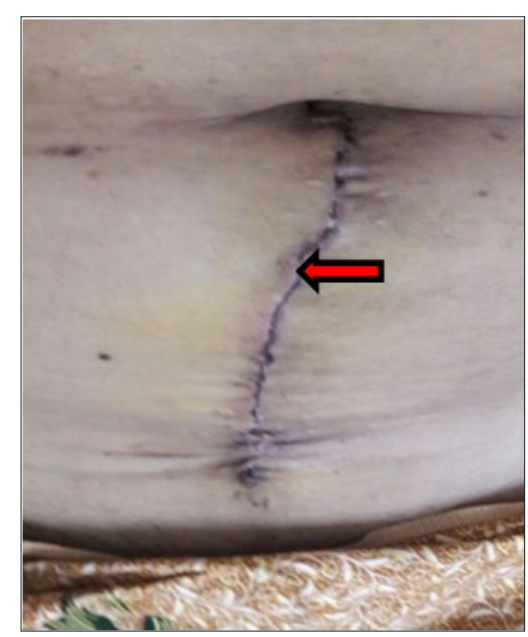

Figure 1: The CT film findings of the patient display two calcification places.

A reassessment was done at our centre. She was noted to have a raised, non-mobile, dark brown pigmented lesion measuring approximately $3 \times 1 \mathrm{~cm}$ at the lower part of her old laparotomy scar (Figure 1). She had delicate point of tenderness to palpation over the raised dark brown lesion. The trans-abdominal ultrasound showed a normal size anteverted uterus with left hydrosalphinx. A pocket of fluid collection was also noted beneath the pigmented lesion. She was posted for diagnostic laparoscopy, adhesiolysis, bilateral salphingectomy and excision of possible stitch granuloma under general anaesthesia.Intra-operatively, there were multiple adhesions between the uterus, fallopian tubes and the bowel. Adhesiolysis and bilateral salphingectomy were done and both Fallopian tubes sent for histopathological examination. The old lower midline scar and the lesion were explored. The lesion contained a cavity that breaches and extends to the rectus sheath. Hemoserous, non foul smelling fluid was drained from the cavity. The scar was completely excised with the granuloma portion and sent for histopathological evaluation (Figure 2).The incision wound was closed by subcuticular method using monosyn $3 / 0$.
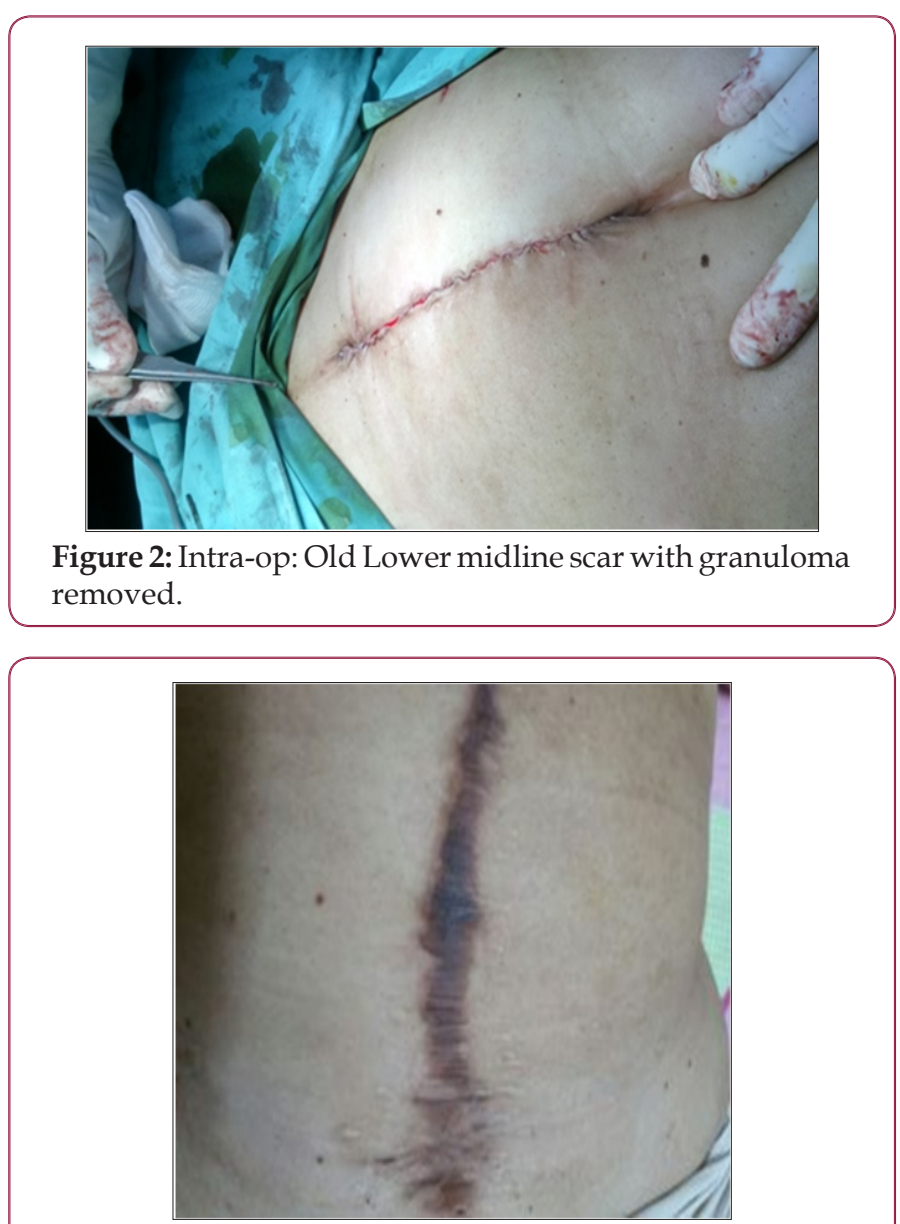

Figure 3: 9 months post-surgery.

Her post-operative recovery was unremarkable. She was discharged on day 3 post surgery. A single dose of GnRH analogue was administered prior to discharge. We continued to review her at our out-patient clinic. At 9 months post-surgery, the wound was clean and well healed (Figure 3). Her pain has subsided and she no longer experience discharge from the surgical scar wound. She started to regain her normal menstruation and is currently undergoing IVF treatment at a fertility centre.The histopathological reports showed mild chronic salphingitis of the right Fallopian tube, whilst the left Fallopian tube was consistent with chronic salphingitis with foci of endometriosis. The sample from the excised scar showed foreign body granuloma with endometriosis. This is also known as cutaneous endometriosis. Microscopically, the section showed skin with the underlying dermis and subcutaneous tissue. A cystic cavity was noted in the dermis lined by flattened epithelium surrounded by haemosiderin laden macrophages and dense lymphoplasmacytic cells (Figure4). There were suture materials surrounded by multinucleated giant cells of foreign body types present (Figure 5). Few islands of endometrial glands and stroma were noted within the stroma. 


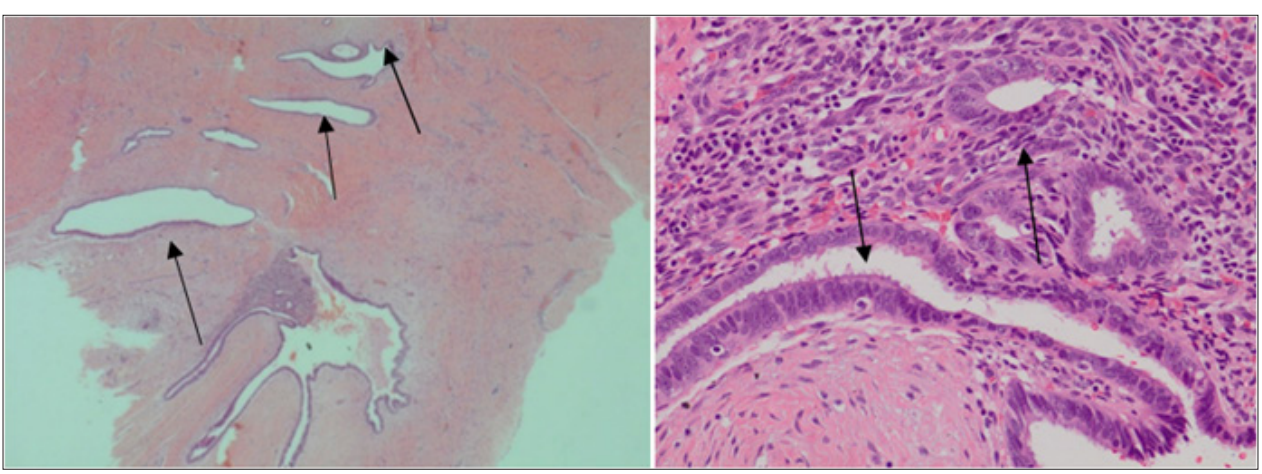

Figure 4: A: Magnified x 4: A few endometrial glands and stroma in the underlying fibrocollagenous tissue stroma. B: Magnified $x$ 20: A few endometrial glands surrounded by endometrial stroma.

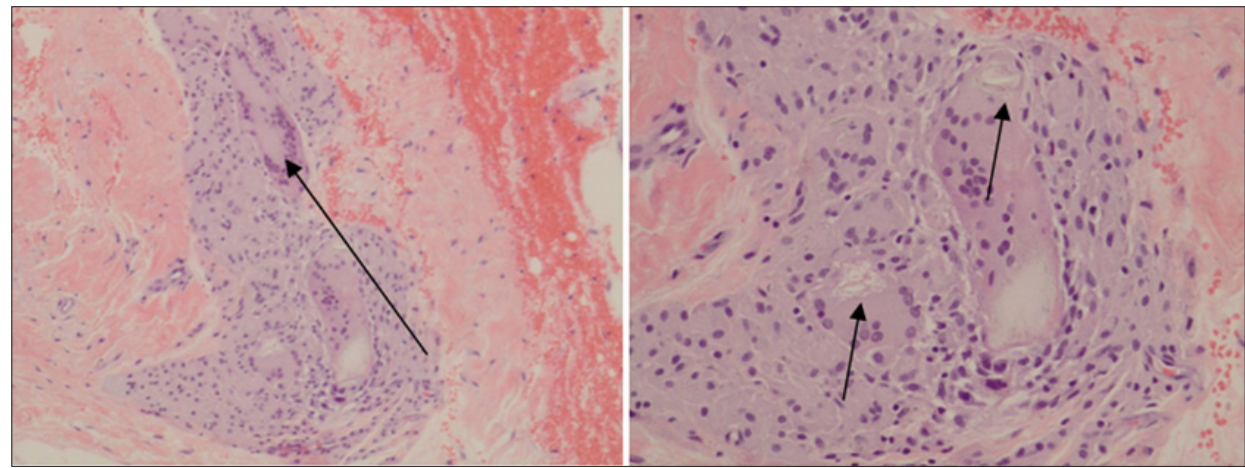

Figure 5: A: Magnified x 10: A few multinucleated giant cells of foreign body types, B: Magnified x 20: Suture material surrounded by multinucleated giant cells of foreign body type.

\section{Discussion}

The diagnosis of scar endometriosis may be difficult and challenging. Clinically the characteristics of scar endometriosis include lumps or nodule over the scar site that usually associates with pain and gradual increasing in size, bleeding or discharge from the lesion and skin discolouration. Only $20 \%$ of the patients presents with cyclical manifestation of symptoms with menstruation. Most patients would usually complain of tenderness over a raised, hideous hyperthrophic scar which can be easily misdiagnosed as infected wound or keloid.In order to provide a rapid and accurate pre-operative diagnosis, fine needle aspiration (FNAC) can be employed to determine the nature of the lesion. It can be done over the lesion which classically appears as a firm nodule. This will help in differentiating it from other possible lesions such as metastatic disease, desmoid tumor, lipoma, sarcoma, cysts, nodular and proliferative fasciitis, fat necrosis, hematoma or abscesss $[9,10]$.

Imaging studies are only useful in determining the extent of the disease and planning of operative resection. This is especially useful in recurrent and large lesions. However, these modalities are non-specific and there are insufficient data available to suggest the best imaging tool over the other.Both surgical excision and medical treatment are part of the management of scar endometriosis. Hormonal suppression with oral contraceptives, progestogen and GnRH analogues have shown to alleviate symptoms. However, hormonal suppression provides temporally relive and recurrence is common after therapy cessation[11]. Therefore, it is strongly recommended that complete surgical excision of the lesion remains as the gold-standard of treatment.

\section{Conclusion}

Scar endometriosis is an uncommon and is often a subtle diagnosis. A Proper detailed history and thorough physical examination should always be performed. Thus, any women presenting with recurrent pain or discharge at an abdominal incision site, especially following gynaecological pelvic surgery with underlying endometriosis should be suspected to have scar endometriosis as part of the differential diagnosis. These women are ideally referred to a tertiary centre for further assessment and management to reduce morbidity and improve quality of life. Surgical excision should be the primary treatment with hormonal suppression as adjunct therapy.

\section{References}

1. Kennedy S, Bergqvist A, Chapron C, D'Hooghe T, Dunselman G, et al. (2005) ESHRE Guideline for The Diagnosis and Treatment of Endometriosis. Hum Reprod 20: 2698-2704

2. Bulletti C, Coccia ME, Battistoni S, Borini A (2010) Endometriosis and Infertility. J Assist Reprod Genet 27(8): 441-447.

3. Culley L, Law C, Hudson N, Denny E, Mitchell H, et al. (2013) The social and psychological impact of endometriosis on women's lives: A critical narrative review. Human Reproduction Update 19(6): 625-639. 
4. (2010) American College of Obstetricians and Gynecologists 2010, Management of endometriosis (Practice Bulletin No.114): News release. Obstetrics \& Gynecology 116(1): 223-236.

5. Steck WD (1965) Cutaneous Endometriosis. JAMA 191: 167.

6. Albrecht LE, Tron V, Rivers JK (1995) Cutaneous endometriosis. International Journal of Dermatology 34(4): 261-262.

7. Francica G, Giardiello C, Angelone G, Cristiano S, Finelli R, et al. (2003) Abdominal wall endometriosis near cesarean delivery scars. J Ultrasound Med 22: 1041-1047.

8. Kaloo P, Reid G, Wong F (2002) Caesarean section scar endometriosis: Two cases of recurrent disease and a literature review. Aust NZ. J Obstet Gynaecol 42: 218-220.

\section{ISSN: 2574-1241}

DOI: 10.26717/BJSTR.2018.06.001397

Ahmad Hazim Wan Ghazali. Biomed J Sci \& Tech Res

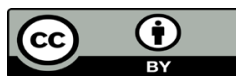

This work is licensed under Creative Commons Attribution 4.0 License

Submission Link: https://biomedres.us/submit-manuscript.php
9. Pathan SK, Kapila K, Haji BE, Mallik MK, Al-Ansary TA, et al. (2005) Cytomorphological spectrum in scar endometriosis: A study of eight cases. Cytopathology 16: 94-99.

10. Catalina-Fernández I, López-Presa D, Sáenz-Santamaria J (2007) Fine needle aspiration cytology in cutaneous and subcutaneous endometriosis. Acta Cyto 151: 380-384.

11. Schoelefield HJ, Sajjad Y, Morgan PR (2002) Cutaneous endometriosis and its association with caesarean section and gynaecological procedures. J Obstet Gynaeco 122: 553-554.

Assets of Publishing with us
BIOMEDICAL
RESEARCHES

